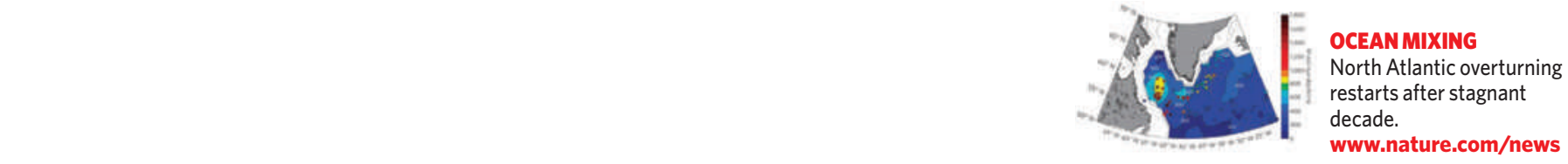

\title{
Can triniobium tin shrink accelerators?
}

The superconducting cavities that drive most of the world's particle accelerators are running out of room for improvement. But new theoretical work suggests that overlooked superconducting materials could be used to make cavities that accelerate particles to higher energies over shorter distances - thereby doing the job more cheaply.

Still, it would take years to develop and test new accelerator components made of materials such as triniobium tin.

For decades, researchers have worked to improve the performance of superconducting cavities. When stacked in a row and pumped with microwave pulses, they build up large electromagnetic fields that accelerate the charged particles used in particle-physics experiments, or in synchrotrons working as high-intensity light sources. The cavities are typically made of niobium, a metal that superconducts when cooled to a few degrees above absolute zero.

\section{Slow and steady}

Scientists have also grappled to improve the performance of the metal, working with different welds, rolls, cavity shapes and surface treatments to make steady but slow gains to the accelerating gradients. The gradients, measured in megaelectronvolts $(\mathrm{MeV})$ per metre, are a measure of how quickly cavities can push particles up to a particular speed. The best laboratory performance of a single cavity is $59 \mathrm{MeV}$ per metre; full machines rely on strings of hundreds of the cavities and would be hard-pressed, under real-world conditions, to achieve gradients half as good.

Other superconducting metals, such as magnesium diboride and triniobium tin, have been discovered, but there have been few efforts to make cavities with them. "People have been assuming that these new materials would be much worse, or not much better than niobium," says James Sethna, a condensedmatter theorist at Cornell University in Ithaca, New York. "Our estimates suggest that you could do dramatically better."

Sethna's work, done with Gianluigi Catelani of Rutgers University in Piscataway, New

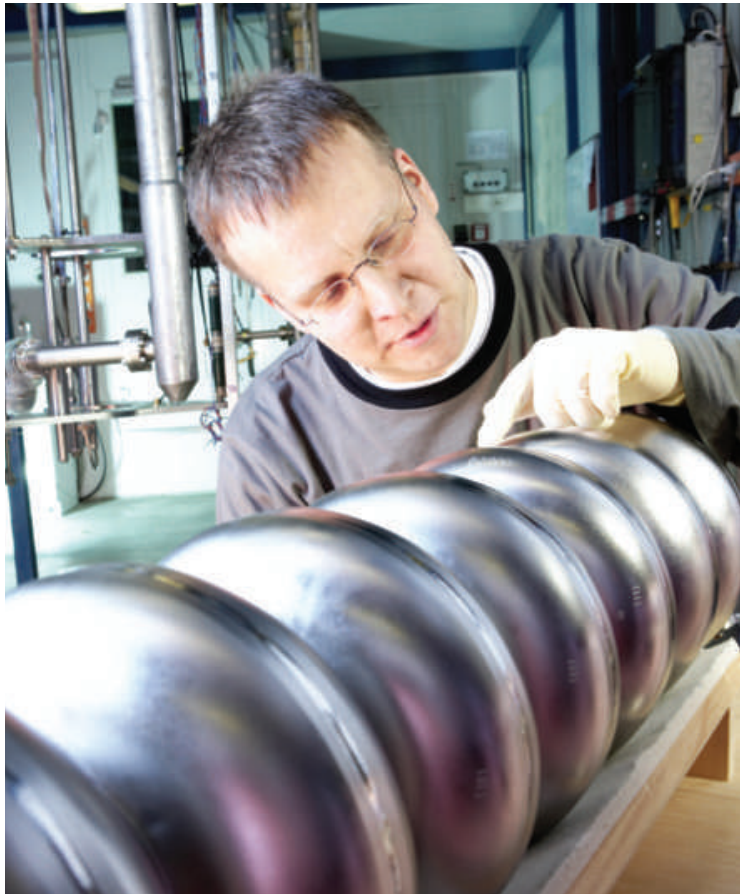

Particle-accelerator cavities are currently made from niobium.
Jersey, will appear in Physical Review B and is available on the physics preprint server arXiv (G. Catelani and J. P. Sethna http://arXiv.org/ abs/0810.4720; 2008). Sethna says that the peak gradient for triniobium tin is $120 \mathrm{MeV}$ per metre and magnesium diboride could reach $200 \mathrm{MeV}$ per metre.

Although a distant goal, achieving such gradients could result in huge savings for future accelerators. For example, the US\$7-billion International Linear Collider (ILC) - a wished for next-generation particle accelerator — will need thousands of cavities, stretching along a tunnel 31 kilometres long, to help it produce energies of 500 gigaelectronvolts. And that's if its niobium cavities can reach ambitious target gradients of a bit more than $30 \mathrm{MeV}$ per metre (see graph). Ramping up the gradients to a theoretically possible peak of $200 \mathrm{MeV}$ per metre could significantly reduce the length of the ILC, therefore also reducing costs of most of its physical parts such as tunnels and beamlines.

\section{Breaking the limits}

According to Sethna, old theories describing the limits of superconducting materials didn't correctly account for ultracold operating temperatures or vortices in the superconductors, which, in creating barriers to the magnetic field lines, allow the cavities to operate at higher gradients. "The excuse has been, 'We've worked so hard on niobium, why try these other materials?'” says Sethna. "All of a sudden, we're telling them, 'you've been listening to the wrong theorists."

Hasan Padamsee, a superconductivity expert at Cornell University, is excited by Sethna's work. In the late 1990s he made a test cavity out of triniobium tin, and it performed less well than niobium alone. But the new work gives Padamsee confidence that the result was due to a problem with the particular sample rather than a fundamental quality of the material.

He says a good working cavity with the new materials would take at least five years to perfect. That's too long to help the ILC in the near term, but perhaps soon enough to be incorporated into upgrades to it.

Peter Kneisel, a senior staff scientist at the Thomas Jefferson National Accelerator Facility in Newport News, Virginia, is less optimistic. He would prefer to wring incremental advances out of niobium cavities, and accept their limitations.

"The grass on the other side of the fence is always greener," says Kneisel, who has spent 40 years working to improve niobium. "It will take, I don't know, 40 years again to bring it to some practical use in accelerators."

Eric Hand 